\title{
Limit theorems for a random walk with memory perturbed by a dynamical system
}

\author{
Cristian F. Coletti * Lucas R. de Lima * ${ }^{*}$ Renato J. Gava ${ }^{\dagger}$ \\ Denis A. Luiz *
}

\begin{abstract}
We introduce a new random walk with unbounded memory obtained as a mixture of the Elephant Random Walk and the Dynamic Random Walk which we call the Dynamic Elephant Random Walk (DERW). As a consequence of this mixture the distribution of the increments of the resulting random process is time dependent. We prove a strong law of large numbers for the DERW and, in a particular case, we provide an explicit expression for its speed. Finally, we give sufficient conditions for the central limit theorem and the law of the iterated logarithm to hold.
\end{abstract}

\section{Introduction}

In this work we introduce a new random walk with memory. The model is obtained as a mixture of two well known random walks, namely the Elephant Random Walk and the Dynamic Random Walk and it is inspired from the theory of Markov switching models where switching among regimes occurs randomly according to a Markov process, see Hamilton [18]. The mixture of models has already been considered in the literature of interacting particle systems. For instance the mixture of spin-flip dynamics and symmetric exclusion processes are known under the name of diffusion-reaction processes, see Belitsky et al. [4] and references there in, and have been intensively studied.

Recently, the Elephant Random Walk (ERW) and other random walks with memory have received considerable attention by many authors, see $[2,3,5-10$, 14,15,21,22,25]. The ERW was introduced by Schütz and Trimper [23] and its dynamic is defined as follows. The elephant starts at the origin and moves one step to the right with probability $q$ and one step to the left with probability

Key words and phrases. Elephant Random Walk, Dynamical System, Strong Law of Large Numbers, Central Limit Theorem.

* Centro de Matemática, Computação e Cognição, Universidade Federal do ABC, Av. dos Estados, 5001, 09210-580 Santo André, São Paulo, Brazil (cristian.coletti@ufabc.edu.br, lucas.roberto@ufabc.edu.br, denis.luiz@ufabc.edu.br).

†Departamento de Estatística, Universidade Federal de São Carlos, Rod. Washington Luiz, km 235, São Carlos, São Paulo, 13565-905, Brazil (gava@ufscar.br) 
$1-q$. At time $n+1$, it chooses at random and with equal probability a number $n^{\prime}$ from the set $\{1, \ldots, n\}$. Then the walker takes, with probability $p$, one step in the same direction of the step given at time $n^{\prime}$ and, with probability $1-p$, it takes one step in the opposite direction. See section 2 for a formal definition of the ERW.

The Dynamic Random Walk (DRW) is a non-homogeneous Markov chain which was introduced by Guillotin-Plantard [16] and whose transition probability of each step is time dependent. In this paper we consider DRW on $\mathbb{Z}$ which evolves in the following manner: at each time a walker takes one step to the right or one step to the left with probability given by a function of the orbit of a given discrete-time dynamical system. See section 2 for a formal definition of the DRW.

This paper is organized as follows. In section 2 we introduce the Dynamic Elephant Random Walk. In section 3 we state our main results and we exhibit some examples where these results hold. In section 4 we prove the strong law of large numbers, the central limit theorem and the law of the iterated logarithm for the Dynamic Elephant Random Walk.

\section{The model}

We begin this section by defining the Elephant Random Walk (ERW) $\left(V_{n}\right)_{n \geq 0}$ with $V_{0}=0$ and increments $\left(W_{n}\right)_{n \geq 1}$. Let $p, q \in[0,1]$, then

$$
\mathbb{P}^{E}\left[W_{1}=1\right]=q, \quad \mathbb{P}^{E}\left[W_{1}=-1\right]=1-q,
$$

and

$$
\mathbb{P}^{E}\left[W_{n}=\eta \mid W_{1}, \ldots, W_{n-1}\right]=\frac{1}{2 n} \sum_{k=1}^{n-1}\left(1+(2 p-1) W_{k} \eta\right) .
$$

The random variables $\left(V_{n}\right)_{n \geq 0}$ and $\left(W_{n}\right)_{n \geq 1}$ are related by the formula $V_{n}=$ $\sum_{i=1}^{n} W_{i}$ where $n \geq 1$. Denote by $p_{n}^{E}($.$) the probability mass function governing$ the law of the increments of the ERW.

Then we define the Dynamic Random Walk (DRW). Let $(\mathcal{X}, \mathscr{A}, \mu, T)$ be a dynamical system where $(\mathcal{X}, \mathscr{A}, \mu)$ is a probability space and $T: \mathcal{X} \rightarrow \mathcal{X}$ is a $\mu$-invariant transformation, i.e., $\mu(A)=\mu(T(A))$ for all $A \in \mathscr{A}$. The DRW is the stochastic process $\left(Y_{n}\right)_{n \geq 0}$ with $Y_{0}=0$ and increments $\left(Z_{n}\right)_{n \geq 1}$ defined by

$$
\mathbb{P}_{x}^{D}\left[Z_{n}=1\right]=f\left(T^{n} x\right) \quad \text { and } \quad \mathbb{P}_{x}^{D}\left[Z_{n}=-1\right]=1-f\left(T^{n} x\right)
$$

where $f: \mathcal{X} \rightarrow[0,1]$ is $\mathscr{A}$-measurable. Here, $T^{0}=i d$ and, for $n \geq 1, T^{n}:=$ $T \circ T^{n-1}$. The random variables $\left(Y_{n}\right)_{n \geq 0}$ and $\left(Z_{i}\right)_{i \geq 1}$ are related by the formula $Y_{n}=\sum_{i=1}^{n} Z_{i}$ where $n \geq 1$. Denote by $p_{x, n}^{D}($.$) the probability mass function$ governing the law of the increments of the DRW

Now we introduce the Dynamic Elephant Random Walk (DERW) on $\mathbb{Z}$. Let $(\mathcal{X}, \mathscr{A}, \mu, T)$ and $f: \mathcal{X} \rightarrow[0,1]$ be as in the definition of the DRW. Fix 
$g: R \subset \mathbb{R} \times \mathbb{N} \rightarrow[0,1]$ and $p, q \in[0,1]$. The DERW is the random walk $\left(S_{n}\right)_{n \geq 0}$ with $S_{0}=0$ and increments $\left(X_{n}\right)_{n \geq 1}$ defined by

$$
\mathbb{P}_{x}\left[X_{n}=\eta\right]=g(\alpha, n) p_{n}^{E}(\eta)+(1-g(\alpha, n)) p_{x, n}^{D}(\eta) .
$$

Here $S_{n}=\sum_{i=1}^{n} X_{i}$. We will denote by $\mathbb{E}_{x}$ the operator expectation induced by $\mathbb{P}_{x}$.

\section{Main results}

Let $\left(S_{n}\right)_{n \geq 0}$ be the DERW with increments $\left(X_{n}\right)_{n \geq 1}$. Set $\mathscr{F}_{n}:=\sigma\left\langle X_{1}, \ldots, X_{n}\right\rangle$ with $\mathscr{F}_{0}=\{\emptyset, \mathcal{X}\}$. We will exclude the case where $g(\alpha, 2)=1$ and $p=0$ without further notice, which is technically inconvenient for our results. To avoid cumbersome notation, we will write for short $\alpha_{n}:=g(\alpha, n)$ for a given $\alpha \in \mathbb{R}$. Set

$$
\begin{aligned}
\ell_{\mathrm{inf}}(\alpha):= & \liminf _{n \rightarrow+\infty} g(\alpha, n), \quad \ell_{\mathrm{sup}}(\alpha):=\limsup _{n \rightarrow+\infty} g(\alpha, n), \\
& \text { and } \ell(\alpha):=\lim _{n \rightarrow+\infty} g(\alpha, n), \text { when it exists. }
\end{aligned}
$$

Condition (T). It is convenient to consider the DERW satisfying the following condition:

$$
p \cdot \ell_{\text {sup }}(\alpha)<1,
$$

which will be referred throughout the text as condition $(\mathrm{T})$.

Theorem 1 (Strong Law of Large Numbers). If the DERW satisfies condition (T), then

$$
\frac{S_{n}-\mathbb{E}_{x}\left[S_{n}\right]}{n} \rightarrow 0 \quad \mathbb{P}_{x}-\text { a.s. }
$$

for every $x \in \mathcal{X}$.

It is worth remarking that if $p=1 / 2$ and the limit $\ell(\alpha)$ exists then we may compute the speed of the DERW.

Corollary 2. Let $\left(S_{n}\right)_{n \geq 0}$ be the DERW. If $p=1 / 2$ and $\ell(\alpha)>0$ then, for $\mu$-almost every $x \in \mathcal{X}$,

$$
\lim _{n \rightarrow+\infty} \frac{S_{n}}{n}=(1-\ell(\alpha))\left(2 \mathbb{E}_{\mu}[f \mid \mathcal{I}](x)-1\right) \quad \mathbb{P}_{x}-\text { a.s. }
$$

where $\mathcal{I}$ stands for the $\sigma$-algebra of $T$-invariant sets.

Corollary 2 provides sufficient conditions for the existence of $\lim _{n \rightarrow+\infty} \frac{S_{n}}{n}$. In the following example we provide a method to compute explicitly the speed of the DERW under some mild conditions. 
Example 1. Consider a DERW satisfying condition (T). In view of Birkhoff's Ergodic Theorem, let $\mathcal{E} \subset \mathcal{X}$ be a set of full measure such that for every $x \in \mathcal{E}$,

$$
\lim _{n \rightarrow+\infty} \frac{1}{n} \sum_{k=0}^{n-1} f\left(T^{k} x\right)=\mathbb{E}_{\mu}[f \mid \mathcal{I}](x) .
$$

Assume that, for a given $x \in \mathcal{E}, \ell(\alpha), \lim _{n \rightarrow+\infty} f\left(T^{n} x\right)$ and $\lim _{n \rightarrow+\infty} \frac{S_{n}}{n}\left(\mathbb{P}_{x}-\right.$ a.s. $)$ do exist. From Cesàro Mean Convergence Theorem we have that

$$
\lim _{n \rightarrow+\infty} \frac{1}{n} \sum_{k=1}^{n} f\left(T^{k} x\right)=\lim _{n \rightarrow+\infty} f\left(T^{n} x\right) .
$$

Denote by $S_{x}$ the $\mathbb{P}_{x}$-a.s. limit of $S_{n} / n$. Then, invoking Theorem 1 we get $\lim _{n \rightarrow+\infty} \frac{\mathbb{E}_{x}\left[S_{n}\right]}{n}=S_{x} \mathbb{P}_{x}-$ a.s. It is easy to verify that

$$
\lim _{n \rightarrow+\infty} \mathbb{E}_{x}\left[X_{n}\right]=(2 p-1) \ell(\alpha) S_{x}+(1-\ell(\alpha))\left(2 \mathbb{E}_{\mu}[f \mid \mathcal{I}](x)-1\right),
$$

see equation (4.1). Invoking again Cesàro Mean Convergence Theorem we get

$$
\lim _{n \rightarrow+\infty} \frac{\mathbb{E}_{x}\left[S_{n}\right]}{n}=(2 p-1) \ell(\alpha) S_{x}+(1-\ell(\alpha))\left(2 \mathbb{E}_{\mu}[f \mid \mathcal{I}](x)-1\right) .
$$

Therefore

$$
S_{x}=\frac{1-\ell(\alpha)}{1-(2 p-1) \ell(\alpha)}\left(2 \mathbb{E}_{\mu}[f \mid \mathcal{I}](x)-1\right)
$$

which agrees with the conclusion of Corollary 2 when $p=1 / 2$.

In what follows, we assume that the DERW satisfies (T). Let

$$
a_{n}:=\prod_{j=1}^{n-1}\left(1+\frac{(2 p-1) g(\alpha, j+1)}{j}\right),
$$

and set $\left(A_{n}\right)_{n \geq 1}$ to be the sequence of non-negative constants defined by

$$
A_{n}^{2}:=\sum_{k=1}^{n} \frac{1}{a_{k}^{2}}\left(1-\mathbb{E}_{x}\left[X_{k}\right]^{2}\right)
$$

We state below a version of the Central Limit Theorem for the DERW followed by some variations of the same result.

Theorem 3 (Central Limit Theorem). Let $\left(S_{n}\right)_{n \geq 0}$ be the DERW with $p \leq 3 / 4$ or $\ell_{\text {sup }}(\alpha)<\frac{1}{4 p-2}$. For all $x \in \mathcal{X}$, one has that if $p<1$ and $\ell_{\mathrm{inf}}(\alpha)>0$, then

$$
\frac{S_{n}-\mathbb{E}_{x}\left[S_{n}\right]}{a_{n} A_{n}} \stackrel{\mathcal{D}}{\rightarrow} \mathcal{N}(0,1) .
$$


Corollary 4. Let the hypothesis of $p<1$ and $\ell_{\inf }(\alpha)>0$ in Theorem 3 be replaced by condition ( $\mathrm{T}$ ) jointly with

$\left(D_{1}\right)$ If $p=1$, then $\liminf _{n \rightarrow+\infty} f\left(T^{n}(x)\right)>0$ and $\limsup _{n \rightarrow+\infty} f\left(T^{n}(x)\right)<\frac{1-\ell_{\sup }(\alpha)}{1-\ell_{\text {inf }}(\alpha)}$; and

$\left(D_{2}\right)$ If $\ell_{\mathrm{inf}}(\alpha)=0$, then $\liminf _{n \rightarrow+\infty} f\left(T^{n}(x)\right)>0, \limsup _{n \rightarrow+\infty} f\left(T^{n}(x)\right)<1-p \cdot \ell_{\mathrm{sup}}(\alpha)$, and $\ell_{\sup }(\alpha)<1$.

Then the conclusion of Theorem 3 remains true.

Corollary 5. Let $\left(S_{n}\right)_{n \geq 0}$ be the DERW. If $p=1 / 2$ and $\ell(\alpha)>0$, then, for $\mu$-almost surely $x \in \mathcal{X}$,

$$
\frac{S_{n}-n(1-\ell(\alpha))\left(2 \mathbb{E}_{\mu}[f \mid \mathcal{I}](x)-1\right)}{\sqrt{n\left(1-(1-\ell(\alpha))^{2}\left(4 \mathbb{E}_{\mu}\left[f^{2} \mid \mathcal{I}\right](x)-4 \mathbb{E}_{\mu}[f \mid \mathcal{I}](x)+1\right)\right)}} \stackrel{\mathcal{D}}{\rightarrow} \mathcal{N}(0,1) .
$$

Theorem 6 (Law of Iterated Logarithm). Under the conditions of Theorem 3 or Corollary 4,

$$
\limsup _{n \rightarrow \infty} \frac{\left|S_{n}-\mathbb{E}_{x}\left[S_{n}\right]\right|}{a_{n} A_{n} \sqrt{\log \log \left(A_{n}\right)}}=\sqrt{2} \quad \mathbb{P}_{x}-\text { a.s. }
$$

We also obtain an almost sure convergence result for the DERW in the regime where the central limit theorem does not hold.

Theorem 7. Let $\left(S_{n}\right)_{n \geq 0}$ be the DERW with $p>3 / 4$ and $\ell_{\mathrm{inf}}(\alpha)>\frac{1}{4 p-2}$. Then, for all $x \in \mathcal{X}$, one has that

$$
\frac{S_{n}-\mathbb{E}_{x}\left[S_{n}\right]}{a_{n}} \rightarrow M \quad \mathbb{P}_{x}-\text { a.s. }
$$

where $M$ is a non-degenerated zero mean random variable.

We finish this section with an example of a DERW where the central limit theorem holds.

Example 2. Let $\mathcal{X}=\left\{(x, y) \in \mathbb{R}^{2}: x^{2}+y^{2} \leq 1\right\}$ be endowed with the Lebesgue $\sigma$-algebra $\mathscr{A}$. Denote by $\mu$ the uniform probability measure defined on $\mathscr{A}_{\mid S^{2}}$ where $S^{2}:=\left\{(x, y) \in \mathbb{R}^{2}: x^{2}+y^{2}=1\right\}$. Namely, $\mu(I(t))=\frac{t}{2 \pi} \wedge 1$ for $t \geq 0$ where $I(t):=\{(\cos \beta, \sin \beta): \beta \in[0, t)\}$.

Consider the following system of ordinary differential equations on $\mathcal{X}$

$$
\left\{\begin{array}{l}
\frac{d x}{d t}=-y+b x\left(x^{2}+y^{2}-1\right) \\
\frac{d y}{d t}=x+b y\left(x^{2}+y^{2}-1\right)
\end{array}\right.
$$

Assume that $b<0$ and, for $(x, y) \neq(0,0)$, denote by $\phi_{t}(x, y):=\phi(t, x, y)$ the solutions of (3.3) satisfying $\phi_{0}=I d$. It is easy to verify, by passing to polar coordinates if necessary, that $\phi_{t}: \mathcal{X} \rightarrow \mathcal{X}$ is $\mu$-invariant for any $t \geq 0$. 


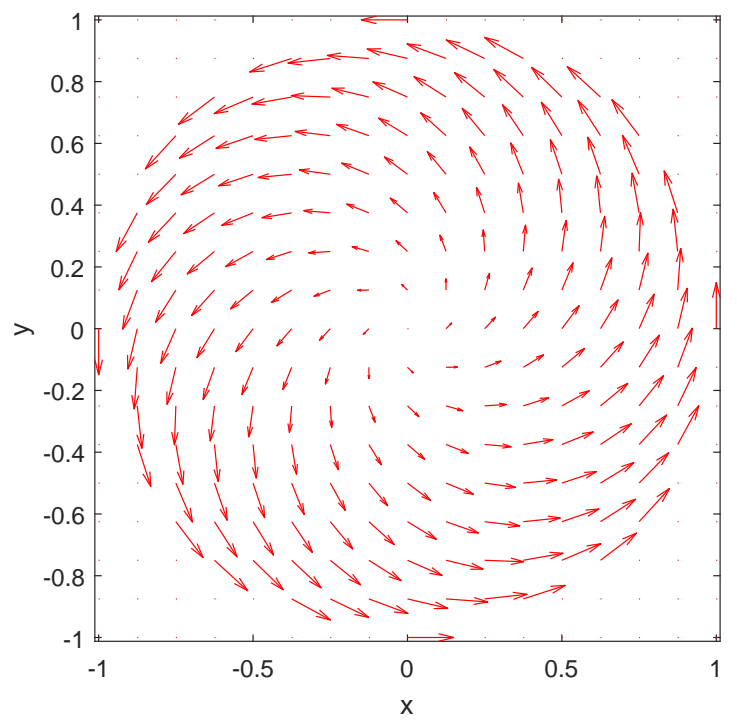

Figure 1: Phase portrait of $\phi_{t}(x, y)$ for $b=-1$

We observe that for any $m, n \geq 0, \phi_{n} \circ \phi_{m}=\phi_{n+m}$ (see [19, p. 175]). Set $T:=\phi_{1}$. In polar coordinates we have that

$$
T(r, \theta)= \begin{cases}\left(\frac{e^{b}}{\sqrt{e^{2 b}+\frac{1}{r^{2}}-1}}, \theta+1\right), & \text { if } 0<r<1 \\ (1, \theta+1), & \text { if } r=1 .\end{cases}
$$

Let $f: \mathcal{X} \rightarrow[0,1]$ be given in polar coordinates by $f(r, \theta)=\cos ^{2} \theta$. It follows from Birkhoff's Ergodic Theorem that

$$
\mathbb{E}_{\mu}[f \mid \mathcal{I}]=\lim _{n \rightarrow+\infty} \frac{1}{n}\left(\sum_{k=1}^{n} \cos ^{2}(\theta+k)\right) \mathbb{1}_{S^{2}}=\frac{1}{2} \mathbb{1}_{S^{2}} \quad \mu-\text { a.s } .
$$

where the $\sigma$-algebra of all $T$-invariant sets $\mathcal{I}$ coincides with $\mathscr{A}$. If $g:[0,1] \times \mathbb{N} \rightarrow$ $[0,1]$ is given by

$$
g(\alpha, n)=\frac{\alpha}{2}+(-1)^{n+1} \frac{\alpha}{n+1},
$$

then $\ell(\alpha)=\frac{\alpha}{2}$. Assuming that $p=1 / 2$ we obtain from Corollary 2 that

$$
\lim _{n \rightarrow+\infty} \frac{S_{n}}{n}=\left(1-\frac{\alpha}{2}\right)\left(\mathbb{1}_{S^{2}}-1\right) \mathbb{P}_{(x, y)}-\text { a.s. }
$$

for every $(x, y) \in \mathcal{X}$. A straightforward computation yields

$$
\mathbb{E}_{\mu}\left[f^{2} \mid \mathcal{I}\right]=\frac{3}{8} \mathbb{1}_{S^{2}} \quad \mu-\text { a.s. }
$$


Thus we may conclude by means of Corollary 5 that

$$
\frac{S_{n}-n\left(1-\frac{\alpha}{2}\right)\left(\mathbb{1}_{S^{2}}-1\right)}{\sqrt{n\left(1+\left(1-\frac{\alpha}{2}\right)^{2}\left(1-\frac{1}{2} \mathbb{1}_{S^{2}}\right)\right)}} \stackrel{\mathcal{D}}{\rightarrow} \mathcal{N}(0,1)
$$

for all $(x, y) \in \mathcal{E}$, where $\mathcal{E}$ is as in (3.1). Hence, if $(x, y) \in \mathcal{E} \backslash S^{2}$, then

$$
\frac{S_{n}+n\left(1-\frac{\alpha}{2}\right)}{\sqrt{n}} \stackrel{\mathcal{D}}{\rightarrow} \mathcal{N}\left(0,1+\left(1-\frac{\alpha}{2}\right)^{2}\right)
$$

Moreover, if $(x, y) \in \mathcal{E} \cap S^{2}$, then

$$
\frac{S_{n}}{\sqrt{n}} \stackrel{\mathcal{D}}{\rightarrow} \mathcal{N}\left(0,1+\frac{1}{2}\left(1-\frac{\alpha}{2}\right)^{2}\right) .
$$

\section{Proofs}

\subsection{The Strong Law of Large Numbers}

The following equation can be obtained by straightforward computation. It gives us an explicit expression for the conditional expectation with respect to the natural filtration of the increments of the Dynamic Elephant Random Walk.

$$
\mathbb{E}_{x}\left[X_{n+1} \mid \mathscr{F}_{n}\right]=\frac{\alpha_{n+1}(2 p-1)}{n} S_{n}+\left(1-\alpha_{n+1}\right)\left(2 f\left(T^{n+1} x\right)-1\right)
$$

Before proving the Strong Law of Large Numbers, we state the lemma below.

Lemma 8. Assume that the DERW satisfies (T). Then $\left(n / a_{n}\right)_{n \geq 1}$ is nondecreasing and

$$
\lim _{n \rightarrow+\infty} \frac{a_{n}}{n}=0 .
$$

Proof. We begin by observing that

$$
\frac{a_{n}}{n}=\prod_{k=1}^{n-1} \frac{\left.k+(2 p-1) \alpha_{k+1}\right)}{k+1}=\prod_{k=1}^{n-1}\left(1-\frac{1-(2 p-1) \alpha_{k+1}}{k+1}\right) .
$$

Let $b_{n}:=\frac{1-(2 p-1) g(\alpha, n+1)}{n+1} \in[0,1)$. Then, $\lim _{n \rightarrow+\infty} \frac{a_{n}}{n}=0$ if, and only if, $\sum_{n} b_{n}=\infty$. Since the DERW satisfies condition (T), we have that $\sum_{n} b_{n}=\infty$. This finishes the proof of the second statement.

Since $(2 p-1) \alpha_{k} \leq 1$, it follows from equation (4.2) that

$$
\frac{n+1}{a_{n+1}}-\frac{n}{a_{n}}=\frac{n !\left(1-(2 p-1) \alpha_{n+1}\right)}{\prod_{k=1}^{n}\left(k+(2 p-1) \alpha_{k+1}\right)} \geq 0,
$$

which yields the conclusion of the first statement. 
Let $M_{n}$ be the random variable defined by $M_{n}:=\frac{S_{n}-\mathbb{E}_{x}\left[S_{n}\right]}{a_{n}}$ for $n \geq 1$ when $a_{n}>0$. In subsection 4.4 we show that the sequence $\left(\stackrel{a}{n}_{n}\right)_{n}$ is a martingale with respect to the natural filtration.

Let $\left(Y_{n}\right)_{n}$ be the martingale difference sequence associated to $\left(M_{n}\right)_{n}$, i.e., $Y_{1}:=M_{1}$ and $Y_{n}:=M_{n}-M_{n-1}$ for $n \geq 2$. Since $Y_{n}=M_{n}-\mathbb{E}_{x}\left[M_{n} \mid \mathscr{F}_{n-1}\right] \mathbb{P}_{x}-$ a.s., it follows from the linearity of the conditional expectation that

$$
Y_{n}=\frac{X_{n}-\mathbb{E}_{x}\left[X_{n} \mid \mathscr{F}_{n-1}\right]}{a_{n}} \mathbb{P}_{x}-\text { a.s. }
$$

for all $n \geq 1$. Since we have by (4.1) that $\left|\mathbb{E}_{x}\left[X_{n} \mid \mathscr{F}_{n-1}\right]\right| \leq 1 \mathbb{P}_{x}$-a.s., one has, for all $n \geq 1$, that

$$
\left|Y_{n}\right| \leq \frac{2}{a_{n}} \quad \mathbb{P}_{x}-\text { a.s. }
$$

for every $x \in \mathcal{X}$.

We now turn to the proof of Theorem 1 and Corollary 2.

Proof of Theorem 1. Set $W_{n}:=\frac{a_{n}}{n} Y_{n}$. By (4.3), we verify that $W_{n}$ is a $\mathscr{F}_{n^{-}}$ measurable random variable such that $\mathbb{E}_{x}\left[W_{n} \mid \mathscr{F}_{n-1}\right]=\frac{a_{n}}{n} \mathbb{E}_{x}\left[Y_{n} \mid \mathscr{F}_{n-1}\right]=0$ $\mathbb{P}_{x}$ - a.s. Therefore, $\left(W_{n}\right)_{n}$ is a sequence of bounded martingale differences.

Since $\mathbb{E}_{x}\left[W_{n}^{2} \mid \mathscr{F}_{n-1}\right] \leq \frac{4}{n^{2}} \quad \mathbb{P}_{x}-$ a.s. by (4.4), we have that, for all $x \in \mathcal{X}$,

$$
\sum_{j=2}^{\infty} \mathbb{E}_{x}\left[W_{j}^{2} \mid \mathscr{F}_{j-1}\right] \leq 4 \sum_{j=2}^{\infty} \frac{1}{j^{2}}<\infty \quad \mathbb{P}_{x}-\text { a.s. }
$$

Thence, it follows from Theorem 2.7 of [17] that $\sum_{j=1}^{n} \frac{a_{j}}{j} Y_{j}=\sum_{j=1}^{n} W_{j}$ converges $\mathbb{P}_{x}$-almost surely as $n \rightarrow+\infty$.

We apply Lemma 8 and Kronecker's lemma obtaining that

$$
\lim _{n \rightarrow+\infty}\left|\frac{S_{n}-\mathbb{E}_{x}\left[S_{n}\right]}{n}\right|=\lim _{n \rightarrow+\infty}\left|\frac{a_{n} M_{n}}{n}\right|=\lim _{n \rightarrow+\infty}\left|\frac{\sum_{j=1}^{n} Y_{j}}{n / a_{n}}\right|=0 \quad \mathbb{P}_{x}-\text { a.s. }
$$

for all $x \in \mathcal{X}$

To prove Corollary 2 we will use the following result on the expectation of the random walk.

Proposition 9. Let $\left(S_{n}\right)_{n \geq 1}$ be the DERW. Then

$$
\mathbb{E}_{x}\left[S_{n}\right]=a_{n}\left(\alpha_{1}(2 q-1)+\sum_{k=1}^{n} \frac{\left(1-\alpha_{k}\right)\left(2 f\left(T^{k} x\right)-1\right)}{a_{k}}\right)
$$


Proof. Note that $S_{1}=X_{1}$ satisfies (4.5) for $n=1$. Suppose that (4.5) holds for a given $n \in \mathbb{N}$. Then

$$
\begin{aligned}
\mathbb{E}_{x}\left[S_{n+1}\right] & =\mathbb{E}_{x}\left[S_{n}\right]+\mathbb{E}_{x}\left[\mathbb{E}_{x}\left[X_{n+1} \mid \mathscr{F}_{n}\right]\right] \\
& =\mathbb{E}_{x}\left[S_{n}\right] \frac{a_{n+1}}{a_{n}}+\left(1-\alpha_{n+1}\right)\left(2 f\left(T^{n+1} x\right)-1\right) \\
& =a_{n+1}\left(\frac{\mathbb{E}_{x}\left[S_{n}\right]}{a_{n}}+\frac{\left(1-\alpha_{n+1}\right)\left(2 f\left(T^{n+1} x\right)-1\right)}{a_{n+1}}\right) .
\end{aligned}
$$

The conclusion follows by induction on $n$.

Proof of Corollary 2. Since $p=\frac{1}{2}, a_{n}=1$ for all $n \geq 1$. Then it can be computed from (4.5) that

$$
\frac{\mathbb{E}_{x}\left[S_{n}\right]}{n}=\frac{1}{n}\left(\alpha_{1}(2 q-1)+\sum_{k=1}^{n}\left(1-\alpha_{k}\right)\left(2 f\left(T^{k} x\right)-1\right)\right) .
$$

Since $\ell(\alpha)=\lim _{n \rightarrow+\infty} \alpha_{n}$ exists, we have that

$$
\begin{aligned}
\frac{1}{n} \mid \sum_{k=1}^{n}\left(1-\alpha_{k}\right)\left(2 f\left(T^{k} x\right)\right. & -1)-\sum_{k=1}^{n}(1-\ell(\alpha))\left(2 f\left(T^{k} x\right)-1\right) \mid \\
& =\frac{1}{n}\left|\sum_{k=1}^{n}\left(2 f\left(T^{k} x\right)-1\right)\left(\ell(\alpha)-\alpha_{k}\right)\right| \\
& \leq \frac{1}{n} \sum_{k=1}^{n}\left|\ell(\alpha)-\alpha_{k}\right| \rightarrow 0 \quad(\text { as } n \rightarrow+\infty) .
\end{aligned}
$$

It follows from the Birkhoff Ergodic Theorem and the Strong Law of Large Numbers (Theorem 1) that

$$
\begin{aligned}
\lim _{n \rightarrow+\infty} \frac{\mathbb{E}_{x}\left[S_{n}\right]}{n} & =\lim _{n \rightarrow+\infty} \frac{1}{n}\left(\alpha_{1}(2 q-1)+\sum_{k=1}^{n}(1-\ell(\alpha))\left(2 f\left(T^{k} x\right)-1\right)\right) \\
& =(1-\ell(\alpha)) \lim _{n \rightarrow+\infty} \frac{1}{n} \sum_{k=1}^{n}\left(2 f\left(T^{k} x\right)-1\right) \\
& =(1-\ell(\alpha))\left(2 \mathbb{E}_{\mu}[f \mid \mathcal{I}](x)-1\right) \quad \mathbb{P}_{x}-\text { a.s. }
\end{aligned}
$$

for $\mu$-almost every $x \in \mathcal{X}$.

\subsection{The Central Limit Theorem}

Define the sequence of positive terms $\left(B_{n}\right)_{n \geq 1}$ given by $B_{n}^{2}:=\sum_{k=1}^{n} \frac{1}{a_{k}^{2}}$.

Lemma 10. If $p \leq 3 / 4$ or $\ell_{\text {sup }}(\alpha)<\frac{1}{4 p-2}$, then $B_{n}^{2} \nearrow+\infty$. Moreover, if $p>3 / 4$ and $\ell_{\text {inf }}(\alpha)>\frac{1}{4 p-2}$, then $\lim _{n \rightarrow \infty}\left|B_{n}\right|<+\infty$. 
Proof. Let $p \leq 3 / 4$. Then

$$
a_{n} \leq \prod_{j=1}^{n-1}\left(1+\frac{1 / 2}{j}\right) \sim \frac{n^{1 / 2}}{\Gamma(3 / 2)},
$$

which implies that $B_{n} \nearrow+\infty$.

Consider now $p>3 / 4$ and let $c_{n}:=\frac{1}{a_{n}^{2}}$. Then it follows from Raabe criterion [20, p. 285] that $B_{n}^{2} \nearrow+\infty$ when $\limsup _{n \rightarrow \infty} n\left(1-\frac{c_{n+1}}{c_{n}}\right)<1$, which is satisfied with

$$
(4 p-2) \ell_{\sup }(\alpha)<1 \text {. }
$$

In the same fashion, we verify that $\lim _{n \rightarrow+\infty} B_{n}^{2}<+\infty$ if $\liminf _{n \rightarrow \infty} n\left(1-\frac{c_{n+1}}{c_{n}}\right)>1$. Note that this is satisfied when

$$
(4 p-2) \ell_{\inf }(\alpha)>1 .
$$

Proof of Theorem 3. We first examine the asymptotic behaviour of $A_{n}$. It follows from Lemma 12 that there exists $n_{0} \in \mathbb{N}$ and $c \in(0,1)$ such that, if $n>n_{0}$, then $c\left(B_{n}^{2}-B_{n_{0}}^{2}\right) \leq A_{n}^{2}-A_{n_{0}}^{2}$. We apply Lemma 10 to verify that $A_{n} \nearrow+\infty$.

Note that we are under conditions of Theorem 1. Therefore, $\frac{S_{n}}{n}=\frac{\mathbb{E}_{x}\left[S_{n}\right]}{n}+$ $\mathrm{o}(1) \mathbb{P}_{x}-$ a.s. for all $x \in \mathcal{X}$. Then it follows from (4.1) that $\mathbb{E}_{x}{ }^{n}\left[X_{n} \mid \mathscr{F}_{n-1}^{n}\right]=$ $\mathbb{E}_{x}\left[X_{n}\right]+\mathrm{o}(1) \mathbb{P}_{x}-$ a.s. We apply it to (4.3) obtaining

$$
\begin{aligned}
\mathbb{E}_{x}\left[Y_{n}^{2} \mid \mathscr{F}_{k-1}\right] & =\frac{1}{a_{n}^{2}}\left(1-2 \mathbb{E}_{x}\left[X_{n} \mid \mathscr{F}_{n-1}\right] \mathbb{E}_{x}\left[X_{n}\right]+\mathbb{E}_{x}\left[X_{n}\right]^{2}+o(1)\right) \\
& =\frac{1}{a_{n}^{2}}\left(1-\mathbb{E}_{x}\left[X_{n}\right]^{2}+o(1)\right) \quad \mathbb{P}_{x}-\text { a.s. }
\end{aligned}
$$

Thus,

$$
\frac{\sum_{k=1}^{n} \mathbb{E}_{x}\left[Y_{k}^{2} \mid \mathscr{F}_{k-1}\right]}{A_{n}^{2}}=\frac{A_{n}^{2}}{A_{n}^{2}}+\frac{B_{n}^{2}}{A_{n}^{2}} \mathrm{o}(1)=1+\mathrm{o}(1) \quad \mathbb{P}_{x}-\text { a.s. },
$$

since $\limsup _{n \rightarrow+\infty}\left|B_{n}^{2} / A_{n}^{2}\right| \leq 1 / c$.

We may arrive to the desired conclusion applying Corollary 3.1 of [17]. To this end, it suffices to verify that the conditional Lindeberg condition holds. Let $S_{n, i}$ be the martingale array with martingale difference sequence $X_{n, i}=\frac{Y_{i}}{A_{n}}$ and $\mathscr{F}_{n, i}:=\mathscr{F}_{i}$.

Consider $p \geq 1 / 2$. Then $a_{n} \geq 1$ and it follows from (4.4) that $\left|X_{n, i}\right| \leq$ $\frac{2}{A_{n}} \mathbb{P}_{x}-$ a.s. Recall that, under the given conditions, $A_{n} \nearrow+\infty$. Hence, for every fixed $\varepsilon>0, \mathbb{P}_{x}\left[\left|X_{n, i}\right|>\varepsilon\right]=0$ for sufficiently large $n$.

Let now $p<1 / 2$. Then $a_{j}^{-1} \leq a_{n}^{-1}$ for all $j \leq n$ and it is immediate to see that $\left|X_{n, i}\right| \leq \frac{2}{a_{n} A_{n}} \mathbb{P}_{x}-$ a.s. We can easily verify that $\lim _{n \rightarrow+\infty} a_{n} A_{n}=+\infty$ (see 
proof of Thm. 6 for details). Therefore, for all $\varepsilon>0$, one has $\mathbb{P}_{x}\left[\left|X_{n, i}\right|>\varepsilon\right]=0$ for sufficiently large $n$.

Now, fix $\varepsilon>0$. Then

$$
\sum_{i=1}^{n} \mathbb{E}_{x}\left[X_{n, i}^{2} \mathbb{1}_{\left\{\left|X_{n, i}\right|>\varepsilon\right\}} \mid \mathscr{F}_{n, i-1}\right] \rightarrow 0 \quad \mathbb{P}_{x}-\text { a.s. }
$$

which completes the proof.

Proof of the Corollary 5. First, observe that since $p=1 / 2$, we have that $a_{n}=1$ for all $n \geq 1$. We also may conclude that $\mathbb{E}_{x}\left[X_{k} \mid \mathscr{F}_{k-1}\right]=\mathbb{E}_{x}\left[X_{k}\right] \mathbb{P}_{x}-$ a.s and $A_{n}^{2}=\sum_{k=1}^{n}\left(1-\mathbb{E}_{x}\left[X_{k}\right]^{2}\right)=\sum_{k=1}^{n}\left(1-\left(1-\alpha_{k}\right)^{2}\left(2 f\left(T^{k} x\right)-1\right)^{2}\right)$. Consequently,

$$
\frac{M_{n}}{A_{n}}=\frac{\frac{S_{n}}{\sqrt{n}}-\sqrt{n} \frac{\mathbb{E}_{x}\left[S_{n}\right]}{n}}{\sqrt{\frac{1}{n} \sum_{k=1}^{n}\left(1-(1-g(\alpha, k))^{2}\left(2 f\left(T^{k} x\right)-1\right)^{2}\right)}} .
$$

Now, similarly to (4.6), we get

$$
\frac{1}{n} \sum_{k=1}^{n}\left(1-\left(1-\alpha_{k}\right)^{2}\left(2 f\left(T^{k} x\right)-1\right)^{2}\right) \sim \frac{1}{n} \sum_{k=1}^{n}\left(1-(1-\ell(\alpha))^{2}\left(2 f\left(T^{k} x\right)-1\right)^{2}\right) .
$$

Note that

$$
\begin{aligned}
& \frac{1}{n} \sum_{k=1}^{n}\left(1-(1-\ell(\alpha))^{2}\right.\left.\left(2 f\left(T^{k} x\right)-1\right)^{2}\right) \\
&=1-(1-\ell(\alpha))^{2}\left(\frac{1}{n}\left[\sum_{k=1}^{n} 4\left(f\left(T^{k} x\right)\right)^{2}-4 f\left(T^{k} x\right)\right]+1\right) .
\end{aligned}
$$

We can apply Birkhoff Ergodic Theorem in the last equation to conclude that

$$
1-(1-\ell(\alpha))^{2}\left(4 \mathbb{E}_{\mu}\left[f^{2} \mid \mathcal{I}\right]-4 \mathbb{E}_{\mu}[f \mid \mathcal{I}]+1\right) \quad \mu-\text { a.s. }
$$

In particular, $\lim _{n \rightarrow+\infty} \frac{1}{\sqrt{n}} A_{n} \neq 0$.

The desired conclusion follows applying (4.9), Corollary 2 and Theorem 3 to (4.8).

\subsection{The Law of Iterated Logarithm}

Proof of Theorem 6. The law of iterated logarithm for the DERW follows from a application of Theorems 1 and 2 of [24]. We have already shown (4.7).

Define $u_{n}=\sqrt{2 \log \log A_{n}^{2}}$ and $K_{n}=\frac{2 u_{n}}{a_{n} A_{n}}$. Let us write the inequality (4.4) in the following way

$$
\left|Y_{n}\right| \leq \frac{2}{a_{n}}=K_{n} \frac{A_{n}}{u_{n}} \quad \mathbb{P}_{x}-\text { a.s. }
$$


It is clear that $K_{n}$ is $\mathcal{F}_{n-1}$ measurable. In order to get (3.2) we need to show that $K_{n} \rightarrow 0$ as $n \rightarrow \infty$.

Recall that $g(\alpha, 2)<1$. Observe that

$$
a_{k} \geq(1-g(\alpha, 2)) \prod_{i=2}^{k-1}\left(1-\frac{1}{i}\right)=(1-g(\alpha, 2)) \frac{1}{k-1},
$$

which implies that

$$
A_{n}^{2} \leq \sum_{k=1}^{n} \frac{1}{a_{k}^{2}} \leq \frac{1}{(1-g(\alpha, 2))^{2}} \sum_{k=1}^{n}(k-1)^{2} \leq \frac{1}{(1-g(\alpha, 2))^{2}} n^{3} .
$$

In other words, $u_{n} \leq \sqrt{2 \log \log \frac{n^{3}}{1-g(\alpha, 2)}}$.

Let us now take care of $a_{n} A_{n}$. Since there exists $c^{\prime}>0$ such that $B_{n}^{2} / A_{n}^{2}$ is bounded by $1 / c^{\prime}$, we get

$$
\begin{aligned}
a_{n}^{2} A_{n}^{2} & \geq \frac{1}{c^{\prime}} a_{n}^{2} B_{n}^{2}=\frac{1}{c^{\prime}} a_{n}^{2} \sum_{j=1}^{n} \frac{1}{a_{j}^{2}}=\frac{1}{c^{\prime}}\left(1+\sum_{j=1}^{n-1} \frac{a_{n}^{2}}{a_{j}^{2}}\right) \\
& =\frac{1}{c^{\prime}}\left(1+\sum_{j=1}^{n-1} \prod_{i=j}^{n-1}\left(1+\frac{(2 p-1) g(\alpha, i+1)}{i}\right)^{2}\right),
\end{aligned}
$$

which yields

$$
\prod_{i=j}^{n-1}\left(1+\frac{(2 p-1) g(\alpha, i+1)}{i}\right) \geq \prod_{i=j}^{n-1}\left(1-\frac{1}{i}\right)=\frac{j-1}{n-1} .
$$

Thence,

$$
a_{n}^{2} A_{n}^{2} \geq \frac{1}{c^{\prime}}\left(1+\sum_{j=1}^{n-1}\left(\frac{j-1}{n-1}\right)^{2}\right) \geq \frac{n}{6 c^{\prime}} .
$$

Combining (4.11) and (4.10) we are able to show that

$$
K_{n}=\frac{2 u_{n}}{a_{n} A_{n}} \leq 4 \sqrt{\frac{3 c^{\prime} \log \log \left(\frac{n^{3}}{(1-g(\alpha, 2))^{2}}\right)}{n}} \rightarrow 0 \quad \text { as } n \rightarrow \infty,
$$

and the claim (3.2) follows.

Proof of Theorem \%. We first observe that $\lim _{n \rightarrow+\infty} B_{n}<+\infty$ by Lemma 10 .

It follows from (4.4) that $\sum_{k=1}^{n} \mathbb{E}_{x}\left[Y_{k}^{2}\right] \leq 4 B_{n}^{2} \quad \mathbb{P}_{x}-$ a.s.. Then by Theorem 12.1 of [26], for all $x \in \mathcal{X}$,

$$
M_{n}=\sum_{k=1}^{n} Y_{k}=\frac{S_{n}-\mathbb{E}_{x}\left[S_{n}\right]}{a_{n}} \rightarrow M \quad \mathbb{P}_{x}-\text { a.s and in } \mathcal{L}^{2} .
$$


Since $\mathbb{E}_{x}\left[M_{n}\right]=0$ for all $n,\left|\mathbb{E}_{x}[M]\right|=\left|\mathbb{E}_{x}\left[M-M_{n}\right]\right| \leq \mathbb{E}_{x}\left[\left|M-M_{n}\right|\right]$. Then

$$
\left|\mathbb{E}_{x}[M]\right| \leq \mathbb{E}_{x}\left[\left|M-M_{n}\right|^{2}\right]^{1 / 2} \rightarrow 0 \quad \text { as } n \rightarrow+\infty .
$$

Furthermore, since $\left(Y_{n}\right)_{n \geq 1}$ is a bounded martingale difference sequence in $\mathcal{L}^{2}$ and it converges almost surely,

$$
\operatorname{Var}_{x}[M]=\lim _{n \rightarrow+\infty} \operatorname{Var}_{x}\left[M_{n}\right]=\sum_{k=1}^{\infty} \mathbb{E}_{x}\left[Y_{k}^{2}\right]>0 .
$$

This leads us to conclude that $M$ is a non-degenerated zero mean random variable.

\subsection{Auxiliary Results}

Proposition 11. The sequence of random variables $\left(M_{n}\right)_{n \geq 1}$ defines a zero mean martingale.

Proof. The zero mean property being straightforward, we only prove that $\mathbb{E}_{x}\left[M_{n+1}\right.$ $\left.\mid \mathscr{F}_{n}\right]=M_{n}$. Indeed, it follows from equation (4.1) that

$$
\begin{aligned}
\mathbb{E}_{x}\left[M_{n+1} \mid \mathscr{F}_{n}\right] & =\frac{S_{n}-\mathbb{E}_{x}\left[S_{n}\right]}{a_{n+1}}+\frac{\mathbb{E}_{x}\left[X_{n+1} \mid \mathscr{F}_{n}\right]-\mathbb{E}_{x}\left[X_{n+1}\right]}{a_{n+1}} \\
& =\frac{1}{a_{n+1}}\left(S_{n}\left(1+\frac{\alpha_{n}(2 p-1)}{n}\right)-\left(1+\frac{\alpha_{n}(2 p-1)}{n}\right) \mathbb{E}_{x}\left[S_{n}\right]\right) \\
& =\frac{S_{n}-\mathbb{E}_{x}\left[S_{n}\right]}{a_{n}} \mathbb{P}_{x}-\text { a.s. }
\end{aligned}
$$

which finishes the proof.

Lemma 12. Let the $D E R W$ be defined with $p<1$ and $\ell_{\mathrm{inf}}(\alpha)>0$. Then

$$
\liminf _{n \rightarrow+\infty} \operatorname{Var}_{x}\left[X_{n}\right]>0 .
$$

Futhermore, if the DERW jointly satisfies $(\mathrm{T}),\left(\mathrm{D}_{1}\right)$, and $\left(\mathrm{D}_{2}\right)$, then $(4.12)$ still holds.

Proof. Note that (4.12) is equivalent to $\limsup _{n \rightarrow+\infty}\left|\mathbb{E}_{x}\left[X_{n}\right]\right|<1$. Since $\frac{S_{n-1}}{n-1} \in$ $[-1,1]$, it follows from (4.1) that conditions

$$
\begin{array}{r}
\liminf _{n \rightarrow+\infty}\left(\alpha_{n}(1-p)+\left(1-\alpha_{n}\right) f\left(T^{n}(x)\right)\right)>0 \\
\limsup _{n \rightarrow+\infty}\left(\alpha_{n} p+\left(1-\alpha_{n}\right) f\left(T^{n}(x)\right)\right)<1
\end{array}
$$

are sufficient to verify (4.12). Moreover,

$$
\begin{array}{r}
(1-p) \ell_{\mathrm{inf}}(\alpha)+\left(1-\ell_{\mathrm{sup}}(\alpha)\right) \liminf _{n \rightarrow+\infty} f\left(T^{n}(x)\right)>0 \\
p \cdot \ell_{\mathrm{sup}}(\alpha)+\left(1-\ell_{\mathrm{inf}}(\alpha)\right) \limsup _{n \rightarrow+\infty} f\left(T^{n}(x)\right)<1
\end{array}
$$


satisfy (4.13) and (4.14), respectively.

Let $p<1$ and $\ell_{\mathrm{inf}}(\alpha)>0$. Then (4.15) is immediately satisfied. Since $f\left(T^{n}(x)\right) \leq 1$, we verify (4.14) by noting that

$$
\limsup _{n \rightarrow+\infty}\left(\alpha_{n} p+\left(1-\alpha_{n}\right) f\left(T^{n}(x)\right)\right) \leq 1-(1-p) \ell_{\inf }\left(\alpha_{n}\right)<1 .
$$

Now, if $p=1$ or $\ell_{\text {inf }}(\alpha)=0$, then (4.15) and (4.16) are straightforwardly satisfied since conditions $(\mathrm{T}),\left(\mathrm{D}_{1}\right)$, and $\left(\mathrm{D}_{2}\right)$ hold.

We finish this section by proving Corollary 4 .

Proof of Corollary 4. The proof follows in the same lines as those of the proof of Theorem 3 and it is an immediate consequence of Lemma 12 and Theorem 1.

\section{Acknowledgements}

C.F.C. thanks FAPESP (grant \#2017/10555-0), L.R.L. thanks FAPESP (grant \#2019/19056-2), and R.J.G. thanks FAPESP (grants \#2017/10555-0 and \#2018/04764-9) for financial support. This study was financed in part by the Coordenação de Aperfeiçoamento de Pessoal de Nível Superior - Brasil (CAPES)

- Finance Code 001.

\section{References}

[1] V. I. Arnold. Ordinary Differential Equations. Springer, 1984.

[2] E. Baur. On a Class of Random Walks with Reinforced Memory, July 2020.

[3] E. Baur and J. Bertoin. Elephant random walks and their connection to pólyatype urns. Phys. Rev. E, 94:052134, Nov 2016.

[4] V. Belitsky, P. A. Ferrari, M. V. Menshikov, and S. Y. Popov. A mixture of the exclusion process and the voter model. Bernoulli, 7(1):119-144, 2001.

[5] I. Ben-Ari, J. Green, T. Meredith, H. Panzo, and X. Tan. Finite-memory elephant random walk and the central limit theorem for additive functionals. Brazilian Journal of Probability and Statistics, (in print).

[6] B. Bercu. A martingale approach for the elephant random walk. Journal of Physics A: Mathematical and Theoretical, 51(1):015201, nov 2017.

[7] B. Bercu, M.-L. Chabanol, and J.-J. Ruch. Hypergeometric identities arising from the elephant random walk. Journal of Mathematical Analysis and Applications, 480(1):123360, 2019.

[8] B. Bercu and L. Laulin. On the center of mass of the elephant random walk, 2019.

[9] B. Bercu and L. Laulin. On the multi-dimensional elephant random walk. Journal of Statistical Physics, 175(6):1146-1163, 2019.

[10] J. Bertoin. Universality of noise reinforced brownian motions, 2020. 
[11] C. F. Coletti, R. Gava, and G. M. Schütz. Central limit theorem and related results for the elephant random walk. J. Math. Phys., 58(5):053303, 8, 2017.

[12] C. F. Coletti, R. Gava, and G. M. Schütz. A strong invariance principle for the elephant random walk. Journal of Statistical Mechanics: Theory and Experiment, 2017(12):123207, dec 2017.

[13] C. F. Coletti, R. J. Gava, and L. R. de Lima. Limit theorems for a minimal random walk model. Journal of Statistical Mechanics: Theory and Experiment, 2019(8):083206, aug 2019.

[14] M. da Silva, E. Rocha, J. Cressoni, L. da Silva, and G. Viswanathan. Non-lévy stable random walk propagators for a non-markovian walk with both superdiffusive and subdiffusive regimes. Physica A: Statistical Mechanics and its Applications, 538:122793, 2020.

[15] G. Di Molfetta, D. O. Soares-Pinto, and S. M. D. Queirós. Elephant quantum walk. Phys. Rev. A, 97:062112, Jun 2018.

[16] N. Guillotin-Plantard. Asymptotics of a dynamic random walk in a random scenery. I. Law of large numbers. Ann. Inst. H. Poincaré Probab. Statist., 36(2):127-151, 2000.

[17] P. Hall and C. C. Heyde. Martingale limit theory and its application. Academic Press, Inc. [Harcourt Brace Jovanovich, Publishers], New York-London, 1980. Probability and Mathematical Statistics.

[18] J. D. Hamilton. A new approach to the economic analysis of nonstationary time series and the business cycle. Econometrica: Journal of the Econometric Society, pages 357-384, 1989.

[19] M. W. Hirsch, R. L. Devaney, and S. Smale. Differential equations, dynamical systems, and linear algebra, volume 60. Academic press, 1974.

[20] K. Knopp. Theory and application of infinite series. Blackie \& Son, 4 edition, 1954.

[21] N. Kubota and M. Takei. Gaussian fluctuation for superdiffusive elephant random walks. J. Stat. Phys., 177(6):1157-1171, 2019.

[22] V. M. Marquioni. Multidimensional elephant random walk with coupled memory. Phys. Rev. E, 100:052131, Nov 2019.

[23] G. M. Schütz and S. Trimper. Elephants can always remember: Exact long-range memory effects in a non-markovian random walk. Phys. Rev. E, 70:045101, Oct 2004.

[24] W. F. Stout. A martingale analogue of Kolmogorov's law of the iterated logarithm. Z. Wahrscheinlichkeitstheorie und Verw. Gebiete, 15:279-290, 1970.

[25] V. H. Vázquez-Guevara. On the almost sure central limit theorem for the elephant random walk. Journal of Physics A: Mathematical and Theoretical, 52(47):475201, oct 2019 .

[26] D. Williams. Probability with martingales. Cambridge Mathematical Textbooks. Cambridge University Press, Cambridge, 1991. 